\title{
High-Bandwidth Organic Photodetector Analyzed by Impedance Spectroscopy
}

\author{
Belen Arredondo, Cristina de Dios, Ricardo Vergaz, Gonzalo del Pozo, Beatriz Romero
}

\begin{abstract}
An organic bulk heterojunction photodetector (OPD) is fabricated on the basis of a blend of poly(3-hexyl thiophene):1-(3-methoxycarbonyl)-propyl-1-1-phenyl-(6,6) C61. The OPD responsivity is calculated from current density-voltage characteristic $(\mathrm{J}-\mathrm{V})$ under green-LED illumination obtaining $1 \mathrm{~A} / \mathrm{W}$. Frequency response at different biases is measured, which shows a high -3-dB cutoff frequency of $343 \mathrm{kHz}$ at $-3 \mathrm{~V}$. Impedance measurements are carried out at different reverse biases, and the results are fitted with a small signal equivalent circuit. The device cutoff frequency $\left(f_{c}\right)$ can be estimated with the parameters extracted from this circuit obtaining good agreement between measured and calculated $\mathbf{f}_{c}$.
\end{abstract}

Index Terms-Bulk heterojunction, cutoff frequency, impedance spectroscopy, organic photodetector.

\section{INTRODUCTION}

O RGANIC bulk heterojunction (BHJ) devices have received great attention due to the low-cost and ease of processing, light weight, mechanical fl xibility and large device areas. BHJ are based on a homogeneous blend of a donor (D) polymer and acceptor (A) molecule forming an interpenetrating network of $\mathrm{D} / \mathrm{A}$ materials with nanoscale heterojunction interface. These BHJ structures ensure that all photogenerated excitons reach a $\mathrm{D} / \mathrm{A}$ interface within the exciton diffusion length, facilitating charge collection at their respective electrodes. Among all possible combinations of D/A pairs, P3HT-PCBM is a well studied system that has been employed in organic solar cells (OSCs) and organic photodetectors (OPDs). This polymer-fullerene blend shows higher hole mobility, improved absorption and higher external quantum eff ciency (EQE) compared to other organic blends [1]. OSCs based on this blend show power conversion eff ciency (PCE) up to $5 \%$ and $6 \%$ for tandem cell geometry [2]-[5]. When biasing in the negative voltage range, the same device structure employed in OSC can operate as OPD. Nevertheless, for detection applications, other characteristic performance different that in solar cells is required. Specifically, quick response times for OPDs are required when

Manuscript received July 3, 2012; revised August 20, 2012; accepted September 3, 2012. Date of publication September 6, 2012; date of current version September 26, 2012. This work was supported by the Comunidad Autónoma de Madrid and Universidad Rey Juan Carlos under Project S2009/ESP-1781 and Project URJC-CM-2010-CET-5173.

B. Arredondo, G. del Pozo, and B. Romero are with the Electronic Technology Department, University Rey Juan Carlos, Móstoles 28933, Madrid, Spain (e-mail: belen.arredondo@urjc.es; gonzalo.delpozo@urjc.es; beatriz.romero@urjc.es).

C. de Dios and R. Vergaz are with the Electronic Technology Department, University Carlos III de Madrid, Leganés 28911, Madrid, Spain (e-mail: cdios@ing.uc3m.es; rvergaz@ing.uc3m.es).

Digital Object Identifie 10.1109/LPT.2012.2217488 employed in a wide range of areas such as military, civil and astronomy applications [6]-[8]. In particular, high speed OPDs for use in optical sensor as well as data transmission systems have received considerable attention [9], [10]. In this context, heterostructure photodetectors based on small molecules and polymer blends with fast response times have been demonstrated [9], [11]-[13]. Besides, the study of carrier dynamic response of organic photodiodes by means of impedance spectroscopy is an important issue that has been subjected to intense research [14], [15]. Impedance spectroscopy is a powerful tool to study dynamics of mobile charge in such devices in light and dark conditions. Moreover, fitti $\mathrm{g}$ impedance measurements with small signal equivalent RC-circuits, circuital and thus material parameters such as mobility and carrier characteristic times can be extracted. However, as far as we are concerned, no previous work has related the OPD frequency response to a modulated input light signal to the parameters extracted from impedance measurements. In this letter we report a high bandwidth OPD with structure ITO/PEDOT:PSS/P3HT:PCBM/Al and cut-off frequency of $343 \mathrm{kHz}$ biasing at $-3 \mathrm{~V}$. Parameters extracted from the fi of an equivalent circuit to impedance measurements have been used to estimate the OPD bandwidth.

\section{FABRICATION AND CHARACTERIZATION}

Patterned indium tin oxide (ITO) glass substrates were cleaned in ultrasonic bath following the typical organic material cleaning process. Substrates were exposed for 30 min to UV irradiation. A hole transport layer of highly conductive poly(3,4-ethylenedioxythiophene)-poly (4-styrene sulfonate) (PEDOT:PSS) from aqueous solution was spincoated and annealed in oven for $30 \mathrm{~min}$. at $110{ }^{\circ} \mathrm{C}$ to obtain a $\sim 40 \mathrm{~nm}$ layer thickness. The active layer was spin-coated from an anhydrous chlorobenzene solution composed by a P3HT:PCBM blend. The P3HT:PCBM ratio was set to 1:0.8 and the solution concentration was 3\% wt. Solutions were stirred for six hours and spin-coated at $4000 \mathrm{rpm}$ to obtain a $80 \mathrm{~nm}$ active layer thickness. Samples were dried in a covered petri dish for $30 \mathrm{~min}$. and baked in oven at $50{ }^{\circ} \mathrm{C}$ for two hours. Al cathode was thermally evaporated on top of the active layer through a shadow mask in an atmosphere of $10^{-6}$ Torr. Finally, devices were encapsulated using a glass cover attached by an epoxy adhesive in order to minimize degradation. All processes were carried out in a glove box in inert atmosphere with low levels of $\mathrm{H}_{2} \mathrm{O}$ and $\mathrm{O}_{2}(<0.1 \mathrm{ppm})$. Device active area is $7 \mathrm{~mm}^{2}$. 


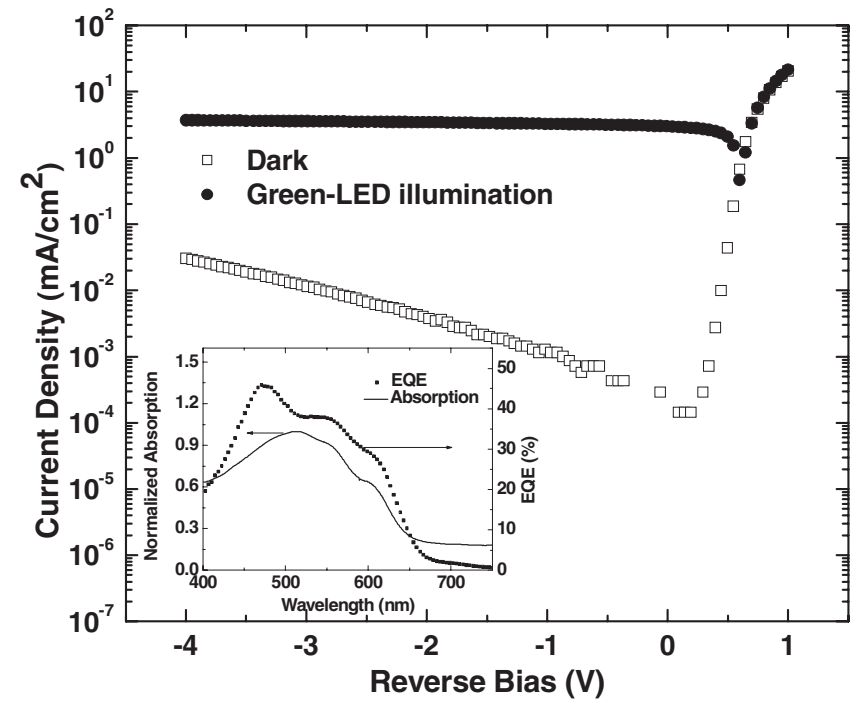

Fig. 1. J-V characteristic. Hollowed symbols: OPD in dark. Filled symbols: under green-LED illumination. Inset shows spectral absorption of a spincoated P3HT:PCBM blend $\mathrm{flm}$ (solid line) and external quantum eff ciency (symbols).

The absorption spectrum was measured with a Varian Cary 500 spectrophotometer. Thickness measurements of the polymeric active layer were performed using a DekTak 150 Veeco profilomete. External quantum efficien y (EQE) was measured with a lock-in SR830, $100 \mathrm{~W}$ halogen tungsten lamp, UV808 Newport UV-enhanced silicon detector and a Thorlabs mechanical chopper.

Current density-voltage characteristics were carried out with a semiconductor parameter analyzer Agilent 4155C and a source generator Agilent 41501B. Cells were illuminated with a green LED Vishay TLLG5400 $(\lambda=530 \mathrm{~nm})$.

The OPD frequency response was evaluated using the same green LED modulating the optical emission using a transconductance amplif er that varied the current f ow through the device. The resulting modulated light illuminated the OPD. The detector under study was reverse biased and connected to an equivalent $100 \Omega$ load; its response has been characterized considering two separate experimental set-ups. The frst one, aimed for frequencies below $100 \mathrm{kHz}$, is based on a high sensitive Lock-in Amplif er (Stanford SRS 830). The second scheme, suitable for frequencies above $60 \mathrm{kHz}$, relies on an electronic spectrum analyzer (ESA Agilent EXA N9010A). This second measurement set-up had to be considered after it was experimentally shown that OPD bandwidth exceeded the capabilities of the lock-in amplifie .

Impedance measurements were carried out with a Solartron 1260 impedance analyzer. The diode was connected to the analyzer that fed the input signal, biasing the diode at different dc levels and superimposing an alternating signal of sweeping frequency with amplitude $100 \mathrm{mV}$.

\section{RESUlTS AND Discussion}

Fig. 1 shows the J-V curves of the OPD in dark and under illumination of a green-LED. The incident power on the OPD is $3.3 \mathrm{~mW} / \mathrm{cm}^{2}$. The dark current density at zero bias is $2.6 \times 10^{-4} \mathrm{~mA} / \mathrm{cm}^{2}$. The photocurrent density under reverse

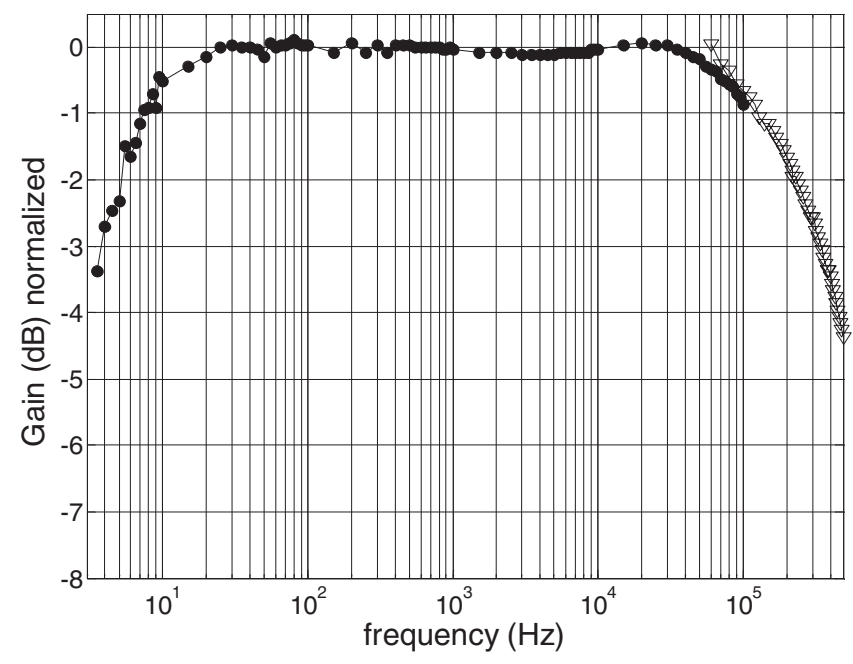

Fig. 2. Optoelectronic small signal frequency response of the OPD biased at $-3 \mathrm{~V}$ under modulated green-LED illumination. Frequency response was characterized with a $100-\mathrm{kHz}$ lock-in amplifie setup (fille circles) and an electronic spectrum analyzer from $60 \mathrm{kHz}$ to $500 \mathrm{kHz}$ (hollowed triangles).

bias maintains in a quasi constant range from $3 \mathrm{~mA} / \mathrm{cm}^{2}$ at zero bias up to $3.7 \mathrm{~mA} / \mathrm{cm}^{2}$ at $-4 \mathrm{~V}$, which is an optimum tendency for detection applications. At forward bias, dark and illuminated currents overlap. The OPD responsivity in the quasi-constant current range is calculated to be approximately $1 \mathrm{~A} / \mathrm{W}$. This value is comparable to that of inorganic devices.

Inset shows absorption spectra of a P3HT:PCBM (1:0.8) fil and the EQE of the device. Absorption and EQE maximums appear at $510 \mathrm{~nm}$ and $470 \mathrm{~nm}$ respectively and secondary shoulders appear at the same wavelengths (558 and $605 \mathrm{~nm})$ [16], [17].

Fig. 2 shows the normalized optoelectronic small signal frequency response of the OPD. Results have been normalized to the maximum gain. The device bandwidth increases with reverse bias, being the $-3 \mathrm{~dB}$ cut-off frequency of $343 \mathrm{kHz}$ at $-3 \mathrm{~V}$. This value for the cut-off frequency is a state-of-theart result being higher than that measured for similar devices [9] and in the same order of magnitude as that of an organic photodetector with inverted structure [12].

Fig. 3 displays the electrical impedance measured in the $1 \mathrm{~Hz}-10 \mathrm{MHz}$ frequency range at different reverse bias. The spectra resemble a depressed semicircle that has been successfully modelled with the equivalent electrical circuit shown in the inset where CPE is a constant phase element with $Z_{\mathrm{CPE}}=\frac{1}{\left(\mathrm{CPE}_{T}\right)(j \omega)^{\left(\mathrm{CPE}_{P}\right)}}$. In frst approximation $\mathrm{CPE}$ is equivalent to a non-ideal capacitance with $\mathrm{CPE}_{\mathrm{T}}$ and $\mathrm{CPE}_{\mathrm{P}}$ being impedance parameters [15]. CPE is an adjustable parameter affected by the device geometry and it takes into account non-homogeneities such as porosities, roughness and surface states. Solid lines in fi . 3 show the ftting results using this circuit and $\mathrm{f} g$. 4 shows the parameters extracted from the $\mathrm{fts}$ at different bias. $\mathrm{CPE}_{\mathrm{P}}$ values range from 0.94 (at $-1 \mathrm{~V}$ ) to 0.96 (at $-3 \mathrm{~V}$ ), as close to 1 that in subsequent analysis CPE will be treated as a capacitor. $\mathrm{CPE}_{\mathrm{T}}$ can be then approximated to a capacitance with values ranging from $3.3 \mathrm{nF}$ (at $-3 \mathrm{~V}$ ) to $4.7 \mathrm{nF}$ (at $-1 \mathrm{~V}$ ). $\mathrm{R}_{\mathrm{s}}$ is the quasi-constant series resistance modelling metallic contacts, wires, etc. $R_{p}$ accounts for the 


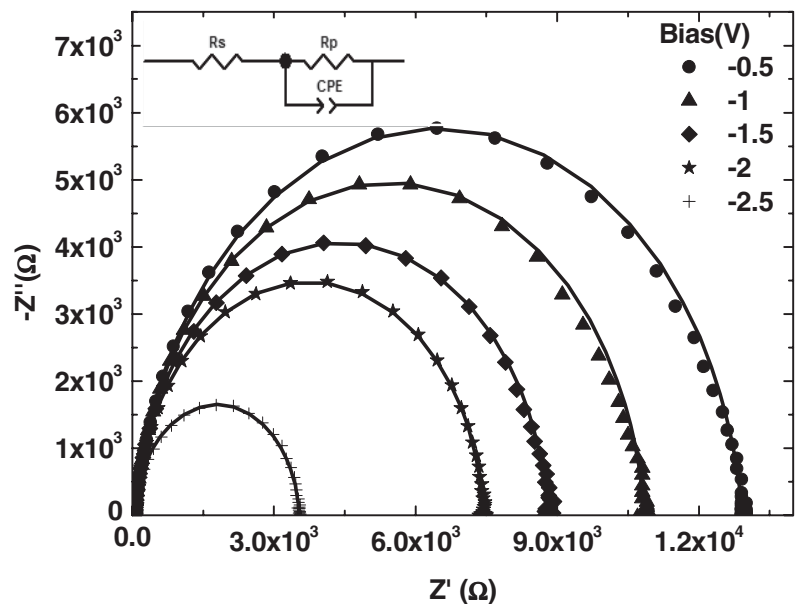

Fig. 3. Impedance spectra measured under illumination conditions of green LED at different bias (symbols). Solid lines show the $\mathrm{ft}$ to the circuit in the inset.

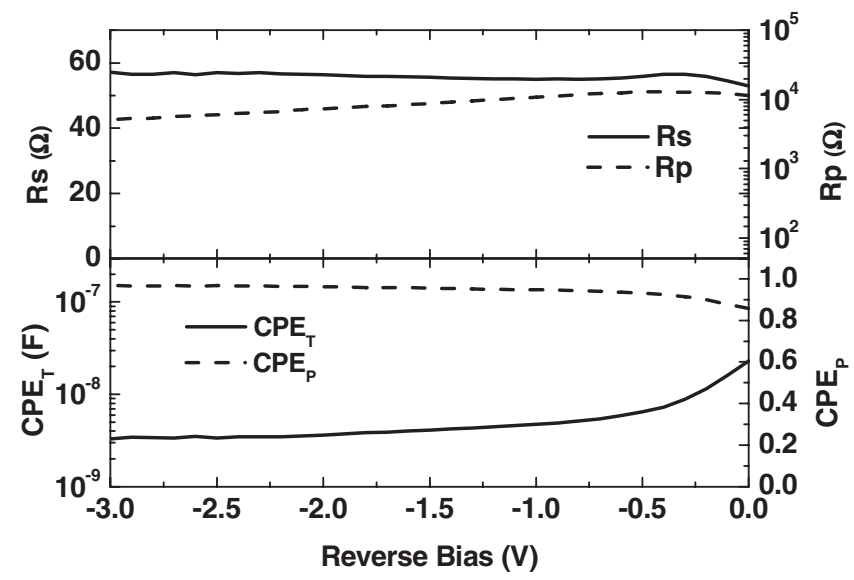

Fig. 4. Evolution with bias of parameters obtained from the fi of Fig. 3.

parallel of the diode dynamical resistance and shunt resistance. In this sense, $R_{p}$ includes both material characteristics such as carrier mobility and leakage currents due to fabrication defects and device geometry. According to García-Belmonte et al. this impedance pattern is typical of systems in which carrier transport is determined by diffusion-recombination between non-absorbing contacts with $\tau_{r e c}=R_{P} C$ being the carrier recombination time [14].

Since both measurements (frequency response and impedance analysis) are performed in the small signal domain, it is possible to make an analogy between the equivalent circuits modelling both of them. From an electrical point of view, equivalent impedance of the circuit shown in the inset of fig 3 can be calculated as:

$$
Z_{T O T}=R_{S}+\frac{R_{P} Z_{C P E}}{R_{P}+Z_{C P E}} \approx\left(R_{S}+R_{P}\right) \frac{1+j \omega \frac{R_{P} R_{S} C}{R_{P}+R_{S}}}{1+j \omega R_{P} C}
$$

where $\mathrm{C}$ corresponds to the parameter $\mathrm{CPE}_{\mathrm{T}}$. The frequency behaviour of this impedance shows a pole at $\omega_{P}=1 / R_{P} C$ that corresponds to the inverse of electron-hole recombination time $\left(\tau_{\text {rec }}\right)$. However, even though carrier transit time may

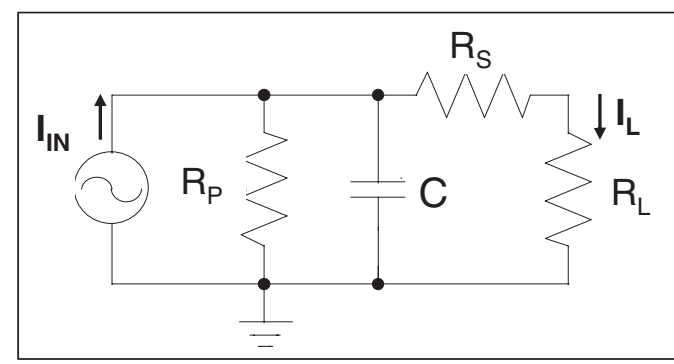

Fig. 5. AC equivalent circuit for the OPD in the cutoff frequency measurement.

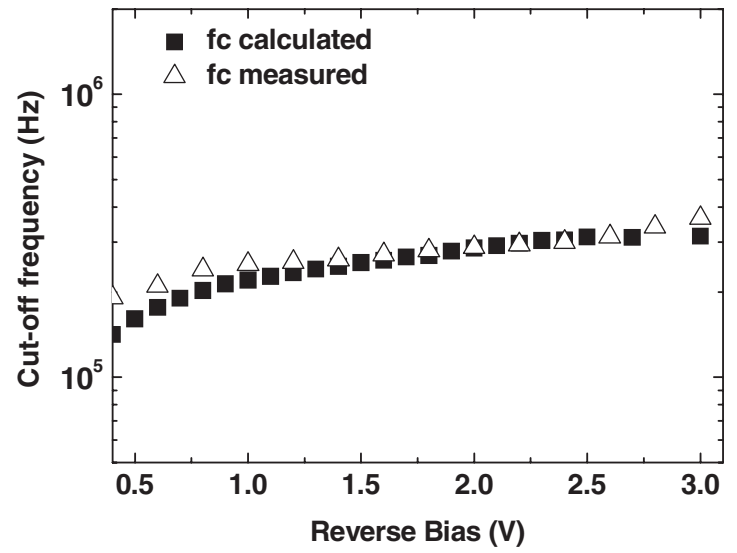

Fig. 6. Hollowed triangles: measured cutoff frequency. Filled squares: calculated cut-off frequency obtained using parameters extracted from the impedance $\mathrm{ft}$ to the electric circuit with $R_{L}=100 \Omega$.

inf uence OPD response, this carrier lifetime cannot be directly related to device cut-off frequency.

Fig. 5 shows the OPD small signal equivalent circuit in the characterization set-up used for retrieving fg. 2 under modulated illumination. This circuit models the OPD electrical behaviour in the bandwidth measurement, with $\mathrm{I}_{\mathrm{IN}}$ being the modulated photogenerated current and $\mathrm{I}_{\mathrm{L}}$ the small signal measured current.

The transfer function of this small signal equivalent circuit is define as:

$\frac{I_{L}}{I_{I N}}=\frac{\left(R_{P} / / Z_{C}\right)}{\left(R_{P} / / Z_{C}\right)+\left(R_{L}^{\prime}\right)}=\frac{R_{P}}{\left(R_{P}+R_{L}^{\prime}\right)\left(1+j \omega \frac{C R_{P} R_{L}^{\prime}}{R_{P}+R_{L}^{\prime}}\right)}$

with $R_{L}^{\prime}=R_{L}+R_{S}$. Equation (2) shows a pole (cut-off frequency) at $\omega_{C}=1 / C\left(R_{P} / / R_{L}^{\prime}\right) \approx 1 / C R_{L}^{\prime}$ for $\left(R_{P}>>R_{L}^{\prime}\right)$.

It is worth mentioning that small signal circuital parameters for the OPD in equations 1 and 2 are the same. Then, from the above calculations it can be inferred that parameters extracted from the fit of impedance measurements can be used to estimate the OPD bandwidth. This can be extremely useful since the equipment needed to measure the OPD response at high frequencies and low signals is not always accessible. Fig. 6 shows a very good agreement between measured and calculated cut-off frequencies at different reverse bias. As mentioned before, cut-off frequency increases with reverse 
bias, obtaining values for measured and calculated $\mathrm{f}_{c}$ of $343 \mathrm{kHz}$ and $315 \mathrm{kHz}$ respectively at $-3 \mathrm{~V}$.

\section{CONCLUSiON}

In summary, we have demonstrated a high bandwidth OPD based on a P3HT:PCBM blend with $\mathrm{f}_{c}$ of $343 \mathrm{kHz}$ at $-3 \mathrm{~V}$. This optoelectronic frequency response suggests that this type of devices could be integrated in applications such as organic high speed optical sensors as well as data transmission systems. Moreover, we have estimated the OPD bandwidth using the parameters extracted from the $\mathrm{ft}$ of impedance measurements with an equivalent circuit. Experimental and calculated $\mathrm{f}_{c}$ show good agreement, demonstrating an easy method to guess the frequency response to a modulated input light signal. This letter underlines the importance to relate impedance and small signal frequency response measurements, being interesting the possibility to apply this technique to any other organic photodetector.

\section{ACKNOWLEDGMENT}

The authors would like to acknowledge Dr. J. Cabanillas for his help with the external quantum eff ciency measurements and R. Criado for his help with automation of frequency response measurements.

\section{REFERENCES}

[1] V. D. Mihailetchi, H. X. Xie, B. de Boer, L. J. A. Koster, and P. W. M. Blom, "Charge transport and photocurrent generation in poly(3hexylthiophene): Methanofullerene bulk-heterojunction solar cells," $A d v$. Funct. Mater, vol. 16, no. 5, pp. 699-708, 2006.

[2] M. Reyes-Reyes, K. Kim, and D. L. Carroll, "High-eff ciency photovoltaic devices based on annealed poly(3-hexylthiophene) and 1-(3-methoxycarbonyl)-propyl-1- phenyl-(6,6)C61 blends," Appl. Phys. Lett., vol. 87, no. 8, pp. 083506-1-083506-3, 2005.

[3] K. Lee, J. Y. Kim, S. H. Park, S. H. Kim, S. Cho, and A. J. Heeger, "Air-stable polymer electronic devices," Adv. Mater, vol. 19, no. 18, pp. 2445-2449, 2007.

[4] G. Li, et al., "High-efficien y solution processable polymer photovoltaic cells by self-organization of polymer blends," Nature Mater., vol. 4 , no. 11, pp. 864-868, 2005.
[5] J. Y. Kim, et al., "Efficien tandem polymer solar cells fabricated by allsolution processing," Science, vol. 317, no. 5835, pp. 222-225, 2007.

[6] F.-C. Chen, S.-C. Chien, and G.-L. Cious, "Highly sensitive, lowvoltage, organic photomultiple photodetectors exhibiting broadband response," Appl. Phys. Lett., vol. 97, no. 10, pp. 103301-103303, 2010.

[7] L. Zhu, Q. Dai, Z.-F. Hu, X.-Q. Zhang, and Y.-S. Wang, "High response organic deep ultraviolet photodetector with PEDOT:PSS anode," Opt. Lett., vol. 36, no. 10, pp. 1821-1823, 2011.

[8] L. Zhu, Q. Dai, Z.-F. Hu, X.-Q. Zhang, and Y.-S. Wang, "Organic deep ultraviolet photodetector with response peak focusing on $270 \mathrm{~nm}$ using the acceptor BAlq," IEEE Photon. Technol. Lett., vol. 23, no. 23, pp. 1835-1837, Dec. 1, 2011.

[9] L. Salamandra, G. Susanna, S. Penna, F. Brunetti, and A. Reale, "Time-resolved response of polymer bulk-heterojunction photodetectors," IEEE Photon. Technol. Lett., vol. 23, no. 12, pp. 780-782, Jun. 15, 2011.

[10] M. Punke, S. Valouch, S. W. Kettlitz, M. Gerken, and U. Lemmer, "Optical data link employing organic light-emitting diodes and organic photodiodes as optoelectronic components," J. Lightw. Technol., vol. 26, no. 7, pp. 816-823, Apr. 1, 2008.

[11] T. Morimune, H. Kajii, and Y. Ohmori, "Photoresponse properties of a high-speed organic photodetector based on copper-phthalocyanine under red light illumination," IEEE Photon. Technol. Lett., vol. 18, no. 24, pp. 2602-2604, Dec. 15, 2006.

[12] D. Baierl, B. Fabel, P. Lugli, and G. Scarpa, "Efficien indium-tin-oxide (ITO) free top-absorbing organic photodetector with highly transparent polymer top electrode," Organic Electron., vol. 12, no. 10, pp. 1669 1673, 2011.

[13] S. Valouch, M. Nintz, S. W. Kettlitz, N. S. Christ, and U. Lemmer, "Thickness-dependent transient photocurrent response of organic photodiodes," IEEE Photon. Technol. Lett., vol. 24, no. 7, pp. 596-598, Apr. $1,2012$.

[14] G. G. Belmonte, A. Munar, E. M. Barea, J. Bisquert, I. Ugarte, and R. Pacios, "Charge carrier mobility and life time of organic bulk heterojunctions analyzed by impedance spectroscopy," Organic Electron., vol. 9 , no. 5, pp. 847-851, 2008.

[15] G. Perrier, R. de Bettignies, S. Berson, N. Lemaitre, and S. Guillerez, "Impedance spectrometry of optimized standard and inverted P3HTPCBM organic solar cells," Solar Energy Mater. Solar Cells, vol. 101, pp. 210-216, Jun. 2012.

[16] V. D. Mihailetchi, H. Xie, B. de Boer, L. J. A. Koster, and P. W. M. Blom, "Charge transport and photocurrent generation in poly(3hexylthiophene): Methanofullerene bulk-heterojunction solar cells," $A d v$. Funct. Mater., vol. 16, no. 5, pp. 699-708, 2006.

[17] K. Kim, J. Liu, M. A. G. Namboothiry, and D. L. Carroll, "Roles of donor and acceptor nanodomains in 6\% eff cient thermally annealed polymer photovoltaics," Appl. Phys. Lett., vol. 90, no. 16, pp. 1635111-163511-3, 2007. 